\title{
DNX-2401, an Oncolytic Virus, for the Treatment of Newly Diagnosed Diffuse Intrinsic Pontine Gliomas: A Case Report
}

\author{
Sonia Tejada ${ }^{1,2,3 *}$, Ricardo Díez-Valle ${ }^{1,2,3}$, Pablo D. Domínguez ${ }^{2,3,4}$, Ana Patiño-García ${ }^{2,3,5}$, \\ Marisol González-Huarriz ${ }^{2,3}$, Juan Fueyo ${ }^{6}$, Cande Gomez-Manzano ${ }^{6}$, Miguel Angel ldoate ${ }^{7}$, \\ Joanna Peterkin ${ }^{8}$ and Marta M. Alonso ${ }^{2,3,5}$ \\ ${ }^{1}$ Department of Neurosurgery, University Hospital of Navarra, Pamplona, Spain, ${ }^{2}$ The Health Research Institute of Navarra \\ (IDISNA), Pamplona, Spain, ${ }^{3}$ Program in Solid Tumors and Biomarkers, Foundation for the Applied Medical Research, \\ Pamplona, Spain, ${ }^{4}$ Department of Radiology, University Hospital of Navarra, Pamplona, Spain, ${ }^{5}$ Department of Pediatrics, \\ University Hospital of Navarra, Pamplona, Spain, ${ }^{6}$ Brain Tumor Center, The University of Texas MD Anderson Cancer Center, \\ Houston, TX, United States, ${ }^{7}$ Department of Pathology, University Hospital of Navarra, Pamplona, Spain, ${ }^{8}$ DNAtrix, Inc., \\ Houston, TX, United States
}

OPEN ACCESS

Edited by:

Jaume Mora,

Hospital Sant Joan de Déu

Barcelona, Spain

Reviewed by: David D. Eisenstat,

University of Alberta, Canada

Carsten Friedrich,

University of Rostock, Germany

${ }^{*}$ Correspondence: Sonia Tejada stejada@unav.es

Specialty section: This article was submitted to

Neuro-Oncology and

Neurosurgical Oncology,

a section of the journal

Frontiers in Oncology

Received: 15 January 2018 Accepted: 22 February 2018

Published: 12 March 2018

Citation:

Tejada S, Díez-Valle R, Domínguez PD, Patiño-García A, González-Huarriz M, Fueyo J, Gomez-Manzano C, Idoate MA,

Peterkin $J$ and Alonso MM (2018) DNX-2401, an Oncolytic Virus, for the Treatment of Newly

Diagnosed Diffuse Intrinsic Pontine Gliomas: A Case Report.

Front. Oncol. 8:61. doi: 10.3389/fonc.2018.00061
Diffuse intrinsic pontine gliomas (DIPGs) are aggressive glial brain tumors that primarily affect children, for which there is no curative treatment. Median overall survival is only one year. Currently, the scientific focus is on expanding the knowledge base of the molecular biology of DIPG, and identifying effective therapies. Oncolytic adenovirus DNX-2401 is a replication-competent, genetically modified virus capable of infecting and killing glioma cells, and stimulating an anti-tumor immune response. Clinical trials evaluating intratumoral DNX-2401 in adults with recurrent glioblastoma have demonstrated that the virus has a favorable safety profile and can prolong survival. Subsequently, these results have encouraged the transition of this biologically active therapy from adults into the pediatric population. To this aim, we have designed a clinical Phase I trial for newly diagnosed pediatric DIPG to investigate the feasibility, safety, and preliminary efficacy of delivering DNX-2401 into tumors within the pons following biopsy. This case report presents a pediatric patient enrolled in this ongoing Phase I trial for children and adolescents with newly diagnosed DIPG. The case involves an 8-year-old female patient with radiologically diagnosed DIPG who underwent stereotactic tumor biopsy immediately followed by intratumoral DNX-2401 in the same biopsy track. Because there were no safety concerns or new neurological deficits, the patient was discharged 3 days after the procedures. To our knowledge, this is the first report of intratumoral DNX-2401 for a patient with DIPG in a clinical trial. We plan to demonstrate that intratumoral delivery of an oncolytic virus following tumor biopsy for pediatric patients with DIPG is a novel and feasible approach and that DNX-2401 represents an innovative treatment for the disease.

Keywords: diffuse intrinsic pontine gliomas, phase I clinical trial, oncolytic virus, delta-24-RGD, DNX-2401, intratumoral, MEMS cannula, biopsy

\section{INTRODUCTION}

Diffuse intrinsic pontine gliomas (DIPGs) are brain tumors located in the pons, the diagnosis of which is based on magnetic resonance imaging (MRI). The 2016 World Health Organization (WHO) classification of Central Nervous System tumors regards these tumors as diffuse midline gliomas H3 K27M-mutants (1). There is no biopsy requirement (2) to confirm tumor histopathology prior 
to initiating currently available treatment which is radiotherapy, with or without chemotherapy (3). However, stereotactic biopsy for pediatric brainstem tumors is associated with a low risk of complications and could be essential for individualizing treatment based on molecular profiling (4). The prognosis for these young patients is dire, with a median overall survival of only 12 months (5).

The lack of effective treatments for DIPG has propelled the field into developing alternative therapeutic strategies that includes biological agents. Oncolytic adenoviruses modified to replicate in, and selectively destroy, tumor cells represents a promising new treatment approach for DIPG. DNX-2401 (formerly Delta-24-RGD) is a replication-competent adenovirus modified to enhance tropism to glioma cells and replicate in cancer cells that have a defective Rb pathway. The virus has already demonstrated safety and efficacy in animal models of gliomas $(6,7)$, adults with recurrent glioblastoma (8), and animal models of DIPG (9).

We have designed an open-label, dose-escalating clinical trial to determine the safety and efficacy (tumor response and survival), of single dose intratumoral DNX-2401 following biopsy for pediatric patients with newly diagnosed DIPG (10). The starting dose of DNX-2401 at $1 \times 10^{10}$ viral particles $(v p)$ is lower than that used in adults $\left(5 \times 10^{10}\right)$ but may be increased to $5 \times 10^{10}$ if no toxicity is reported. Secondary aims are to collect tissue samples and evaluate possible synergy of DNX-2401 with radiotherapy and chemotherapy.

\section{CASE PRESENTATION}

The patient, an otherwise healthy 8-year-old female who presented with vomiting and a 4-month history of gait disturbance, was initially evaluated for food intolerance. Following complaints of diplopia and headache, MRI without gadolinium (gd) enhancement revealed an infiltrating tumor across the entire length of the pons that was hypointense on $\mathrm{T} 1$ and hyperintense on $\mathrm{T} 2$ sequences. The lesion was diagnosed as DIPG (10). The patient met all clinical trial eligibility criteria (EudraCT: 2016-00157733 ), and her parents provided written informed consent for their daughter to participate in the study.

A transcerebellar, transpeduncular frameless navigated biopsy was performed through a suboccipital entry point, using the Brainlab VarioGuide navigation system (Figure 1). This navigated stereotactic system has proven accuracy for brainstem biopsies (11). Stereotactic guidance to localize the biopsy target was based on a preoperative immediate 3T MRI machine
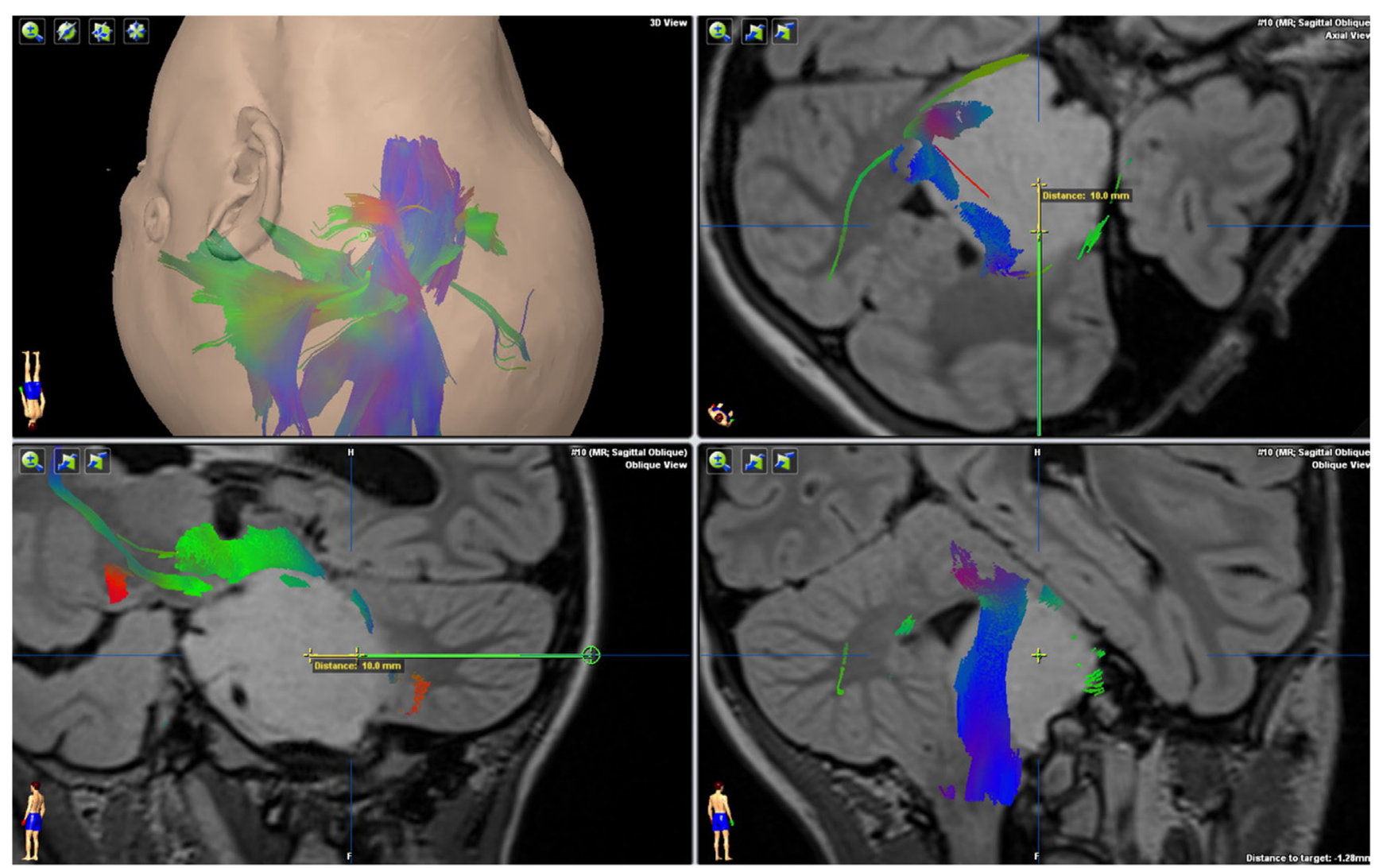

FIGURE 1 | Magnetic resonance imaging T2 FLAIR 3D sequence, with DTI tractography fusion showing the relationship between the tumor and major brain tracts. The green trajectory of the biopsy avoids the corticospinal tract ending in the biopsy target. The yellow segment notes the extra depth (10 mm) of the infusion cannula tip. 
(Skyra, Siemens, Erlangen, Germany). Following withdrawal of the biopsy needle, a cannula specially designed for the delivery of agents into the brain (Alcyone MEMS cannula) was introduced along the same trajectory; however, $10 \mathrm{~mm}$ deeper than the tip of the biopsy needle. The MEMS cannula is a device with two fused microtubes or channels that permit the administration of two independent fluids once inserted into the tissue.

For the patient, one channel was used to infuse $150 \mu \mathrm{L}$ of gd $(2 \mathrm{mM})$ at $0.9 \mathrm{~mL} / \mathrm{h}(10 \mathrm{~min})$ while the second channel was used to deliver $1 \times 10^{10} \mathrm{vp}$ in $1 \mathrm{~mL}$ of DNX-2401 into the tumor at $0.9 \mathrm{~mL} / \mathrm{h}(67 \mathrm{~min})$. The gd was subsequently pushed out of the tumor tissue by the virus as it was infused from the second channel.

An intraoperative MRI was performed immediately after DNX-2401 administration that showed no biopsy complications and gd diffusion through the tumor thereby confirming successful targeted delivery of the virus (Figure 2). The patient tolerated the procedures and was discharged to home from the hospital in $72 \mathrm{~h}$. The pathologist later classified the tumor as a WHO grade II glioma with a H3.3 K27M mutation that was determined by Sanger sequencing (Figure 3). The patient started radiotherapy 2 weeks after the procedure without difficulty.

\section{DISCUSSION}

During the last few decades, DIPG has been considered an inoperable tumor because there have been no benefits gained from biopsy or resection surgery $(12,13)$. However, there has been a resurgence in recent years of the need for performing a biopsy for different reasons: inconclusive images on MRI for atypical lesions (14), the design of clinical trials for DIPG (15), and a significant need to learn more about tumor biology and genetics $(16,17)$.

Although there is a low risk of complications from biopsy (18), the possibility still remains making the procedure difficult to justify outside a clinical trial setting (2). On the other hand, analyzing biopsied tissue will advance our fundamental knowledge of this tumor with the goal of developing new treatments (19) taking intrinsic characteristics such as molecular aberrations and the tumor microenvironment into account. This information has the potential to alter the diagnostic perspective and treatment options to achieve the best patient outcome. Furthermore, tissue analysis will aid the development of reliable transgenic animal models of DIPG (20).

We believe that tumor biopsy followed by targeted therapy in a single surgical session seems to offer the best balance. In this way, the risks of biopsy are offset by the potential therapeutic benefits of novel therapeutics, such as DNX-2401.
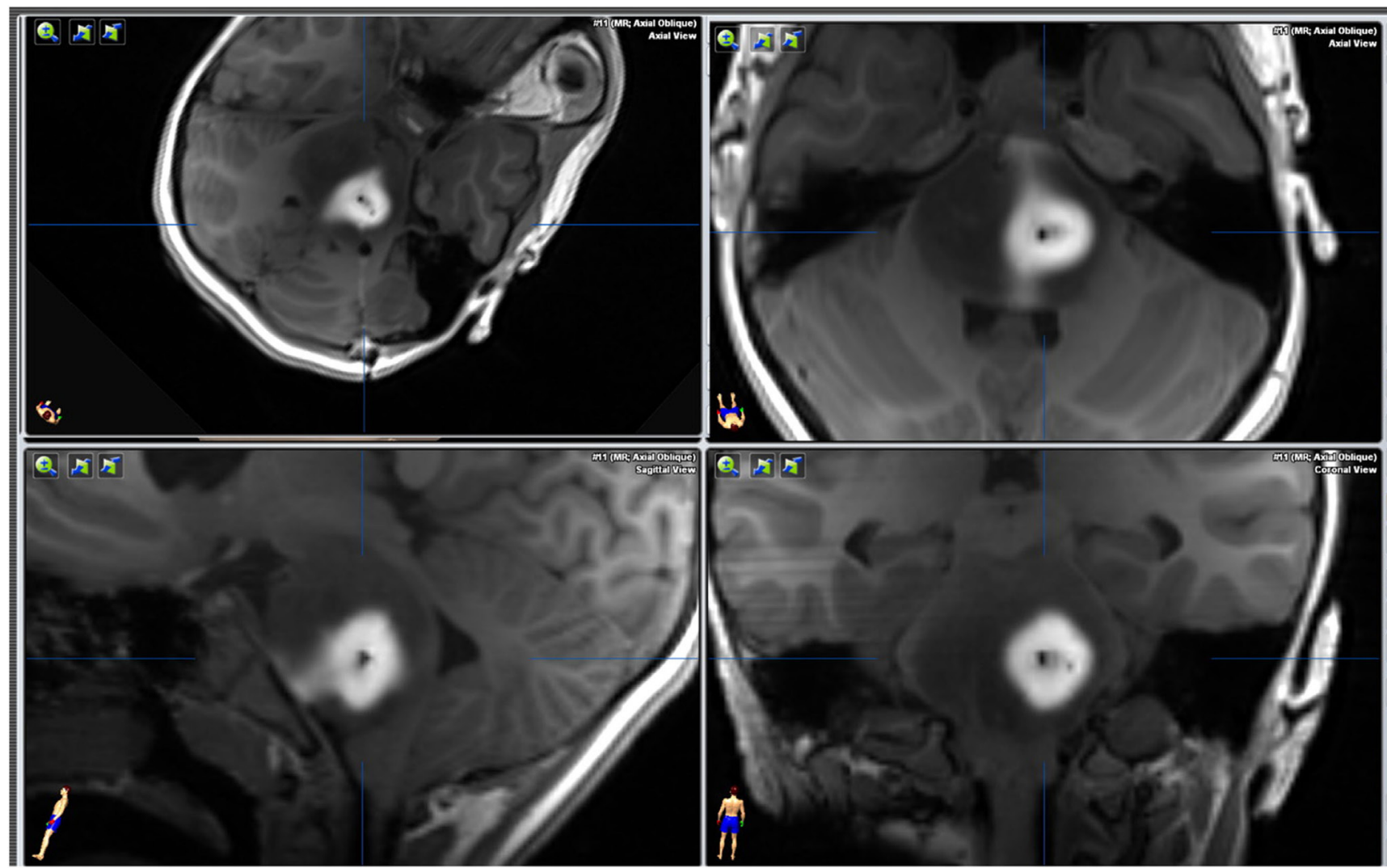

FIGURE 2 | Magnetic resonance imaging T1 GE 3D axial sequence without intravenous contrast, immediately after virus infusion. The intraparenchymal injection of gadolinium before infusing the virus with the MEMS cannula (this cannula has two independent channels) is pushed out from the tumor as the virus is infused from a second channel. 


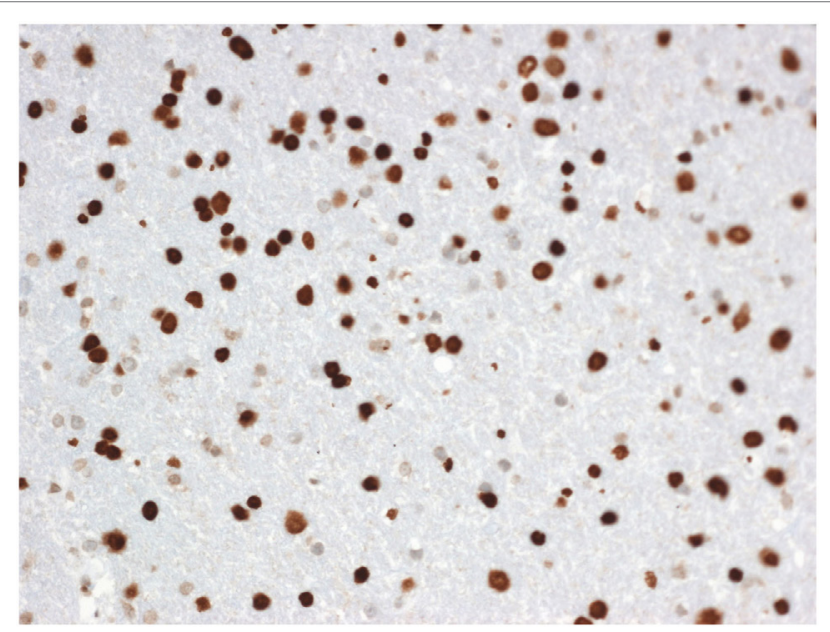

FIGURE 3 | Diffuse midline glioma showing strong nuclear H3K27M mutant protein (immunohistochemistry, X200)

DNX-2401 has shown compelling efficacy in animal models $(21,22)$. The drug has also been assessed in adults with recurrent glioblastoma in several ethics committee-approved clinical trials at different investigative centers including our hospital, the University Hospital of Navarra (EUDRACT number: 2011005935-21). To date, no severe or greater virus-related toxicity has been observed (i.e., no $\geq$ grade 3 in severity per the CTCAE v4.0 toxicity criteria) at a dose of $3 \times 10^{10} \mathrm{vp}$. Median survival has been improved over standard of care including surgery, with some patients surviving three or more years.

DNX-2401 was successfully delivered into DIPG of the first study patient via the MEMS cannula fabricated to deliver fluid into the brain using micro-electro-mechanical systems technology that avoids backflow (23) and confirmed by MRI demonstrating the gd halo. Precise administration into the target lesion increases the potential for an anti-tumor response. In addition, while animal data suggest DNX-2401 followed by radiation, standard therapy for DIPG, improves outcome compared with radiation alone, all patients receive both treatments. As suggested by O'Cathail et al., two treatments given sequentially could be synergistic, leading to a more robust antitumor response (24). This hypothesis also provides a rationale for infusing the virus as first-line therapy before radiation in treatment-naive patients.

Overall, the study patient did not experience any procedurerelated safety issues or virus toxicity during the first 4 weeks following biopsy and virus infusion. Longer term follow-up and the enrollment of additional pediatric patients are necessary to confirm these preliminary results.

\section{CONCLUSION}

This case report describes the first time an oncolytic adenovirus has been injected into DIPG of a pediatric patient in the context of a clinical investigation: a "Phase I trial of DNX-2401 for DIPG for newly diagnosed pediatric patients." Early data generated from this patient's participation in the trial demonstrate biopsy and virus delivered during the same surgical session are both feasible. Additionally, DNX-2401 was well tolerated during the first weeks so that the patient was able to begin radiotherapy 2 weeks later.

We believe the information obtained from this trial could advance the understanding of this devastating disease and facilitate the development of new treatment strategies to alter the current treatment paradigm for DIPG.

\section{ETHICS STATEMENT}

Before initiating the trial, the protocol and all required documents were reviewed and approved by the ethics committee (CEIC) from the Government of Navarra (D24-DIPG) and The Spanish Agency of Medicines and Health Products (AEMPs, Agencia Española del Medicamento y de Productos Sanitarios) Eudra nr 2016-001577-33. Patients younger than 12 years old are not required to sign the informed consent form. Instead, they receive the information by listening to the agreement regarding the treatment, and their capacity to make an informed decision will be confirmed. The patients' parents will then sign the informed consent. Patients older than 12 years old can sign an informed consent along with their parent's signature, and they will receive information according to their comprehension level. Written parental consent was provided by the parents on a consent form approved by the CEIC that described the trial and contained sufficient information to make an informed decision about their daughter's participation in the trial. The designated investigator or research professional obtaining consent also signed the form. Additional written informed consent was obtained from the parents for the publication of this case report.

\section{AUTHOR CONTRIBUTIONS}

MA, ST-S, RD-V, JF, and CG-M designed the study. ST-S and $\mathrm{RD}-\mathrm{V}$ perform surgery and virus delivery. AP and MG-H were in charge of sample collection. AP, MA, and MG-H performed and analyze the molecular study. PD performed and analyze images. ST-S, RD-V, JP, and MA wrote the manuscript. All the authors revised and approved the manuscript. MI performed and analyzed tissue samples, providing the figure and the legend.

\section{ACKNOWLEDGMENTS}

This trial is partially supported by patient donations. We thank DNAtrix for kindly supplying the virus DNX-2401 and Alcyone Lifesciences for donating the Alcyone MEMS cannula and their guidance for improving virus diffusion in the brain. We acknowledge the funding from The Instituto de Salud Carlos III and Fondos Feder Europeos (PI13/125 and PI16/00066 to MA), the Spanish Ministry of Science and Innovation (IEDI-2015-00638 to MA), and the Basque Foundation for Health Research (BIOEF, BIO13/CI/005). Foundation LA CAIXA (to AG and MA), DOD team science award (to MA, JF, and CG-M). 


\section{REFERENCES}

1. Louis DN, Perry A, Reifenberger G, von Deimling A, Figarella-Branger D, Cavenee WK, et al. The 2016 world health organization classification of tumors of the central nervous system: a summary. Acta Neuropathol (2016) 131(6):803-20. doi:10.1007/s00401-016-1545-1

2. Pierre-Aurelien B, Alexandru S, Federico DR, Justyna K, Carmine M, Didier F. Diffuse intrinsic pontine glioma in children: document or treat? World Neurosurg (2016) 93:.e11-4. doi:10.1016/j.wneu.2016.07.011

3. Vanan MI, Eisenstat DD. DIPG in children - what can we learn from the past? Front Oncol (2015) 5:237. doi:10.3389/fonc.2015.00237

4. Hamisch C, Kickingereder P, Fischer M, Simon T, Ruge MI. Update on the diagnostic value and safety of stereotactic biopsy for pediatric brainstem tumors: a systematic review and meta-analysis of 735 cases. JNeurosurg Pediatr (2017) 20(3):261-8. doi:10.3171/2017.2.PEDS1665

5. Kieran MW, Goumnerova LC, Prados M, Gupta N. Biopsy for diffuse intrinsic pontine glioma: a reappraisal. J Neurosurg Pediatr (2016) 18(3):390-1. doi:10.3171/2015.6.PEDS15374

6. Alonso MM, Gomez-Manzano C, Bekele BN, Yung WKA, Fueyo J. Adenovirus-based strategies overcome temozolomide resistance by silencing the O6-methylguanine-DNA methyltransferase promoter. Cancer Res (2007) 67(24):11499-504. doi:10.1158/0008-5472.CAN-07-5312

7. Fueyo J, Alemany R, Gomez-Manzano C, Fuller GN, Khan A, Conrad CA, et al. Preclinical characterization of the antiglioma activity of a tropismenhanced adenovirus targeted to the retinoblastoma pathway. J Natl Cancer Inst (2003) 95(9):652-60. doi:10.1093/jnci/95.9.652

8. Lang FF, Conrad C, Gomez-Manzano C, Yung WKA, Sawaya R, Weinberg JS, et al. Phase I study of DNX-2401 (Delta-24-RGD) oncolytic adenovirus: replication and immunotherapeutic effects in recurrent malignant glioma. J Clin Oncol (2018):JCO2017758219. doi:10.1200/JCO.2017.75.8219

9. Martinez-Velez N, Marigil M, Dominguez P, Aristu J, Fueyo J, GomezManzano C, et al. HG-51delta-24-RDG in combination with radiotherapy for DIPG: opening new therapeutic avenues. Neuro Oncol (2016) 18(Suppl 3): iii58-9. doi:10.1093/neuonc/now073.47

10. Tejada S, Alonso M, Patino A, Fueyo J, Gomez-Manzano C, Diez-Valle R. Phase I trial of DNX-2401 for diffuse intrinsic pontine glioma newly diagnosed in pediatric patients. Neurosurgery (2017). doi:10.1093/neuros/ nyx507

11. Giese H, Hoffmann K-T, Winkelmann A, Stockhammer F, Jallo GI, Thomale U-W. Precision of navigated stereotactic probe implantation into the brainstem. J Neurosurg Pediatr (2010) 5(4):350-9. doi:10.3171/2009.10. PEDS09292

12. Epstein F, McCleary EL. Intrinsic brain-stem tumors of childhood: surgical indications. J Neurosurg (1986) 64(1):11-5. doi:10.3171/jns.1986.64.1.0011

13. Rubin G, Michowitz S, Horev G, Herscovici Z, Cohen IJ, Shuper A, et al. Pediatric brain stem gliomas: an update. Child's Nerv Syst (1998) 14(4-5): 167-73. doi:10.1007/s003810050205
14. Pincus DW, Richter EO, Yachnis AT, Bennett J, Bhatti MT, Smith A. Brainstem stereotactic biopsy sampling in children. J Neurosurg Pediatr (2006) 104(2):108-14. doi:10.3171/ped.2006.104.2.7

15. Roujeau T, Machado G, Garnett MR, Miquel C, Puget S, Geoerger B, et al. Stereotactic biopsy of diffuse pontine lesions in children. J Neurosurg Pediatr (2007) 107(1):1-4. doi:10.3171/PED-07/07/001

16. Broniscer A, Baker JN, Baker SJ, Chi SN, Geyer JR, Morris EB, et al. Prospective collection of tissue samples at autopsy in children with diffuse intrinsic pontine glioma. Cancer (2010) 116(19):4632-7. doi:10.1002/cncr.25405

17. Jones C, Karajannis MA, Jones DTW, Kieran MW, Monje M, Baker SJ, et al. Pediatric high-grade glioma: biologically and clinically in need of new thinking. Neuro Oncol (2017) 19(2):153-61. doi:10.1093/neuonc/now101

18. Puget S, Beccaria K, Blauwblomme T, Roujeau T, James S, Grill J, et al. Biopsy in a series of 130 pediatric diffuse intrinsic pontine gliomas. Childs Nerv Syst (2015) 31(10):1773-80. doi:10.1007/s00381-015-2832-1

19. Infinger LK, Stevenson CB. Re-examining the need for tissue diagnosis in pediatric diffuse intrinsic pontine gliomas: a review. Curr Neuropharmacol (2017) 15(1):129-33.

20. Marigil M, Martinez-Velez N, Domínguez PD, Idoate MA, Xipell E, PatiñoGarcía A, et al. Development of a DIPG orthotopic model in mice using an implantable guide-screw system. PLoS One (2017) 12(1):e0170501. doi:10.1371/journal.pone.0170501

21. Fueyo J, Gomez-Manzano C, Alemany R, Lee PS, McDonnell TJ, Mitlianga P, et al. A mutant oncolytic adenovirus targeting the $\mathrm{Rb}$ pathway produces antiglioma effect in vivo. Oncogene (2000) 19(1):2-12. doi:10.1038/sj.onc.1203251

22. Jiang H, Gomez-Manzano C, Lang FF, Alemany R, Fueyo J. Oncolytic adenovirus: preclinical and clinical studies in patients with human malignant gliomas. Curr Gene Ther (2009) 9(5):422-7. doi:10.2174/156652309789753356

23. Brady ML, Raghavan R, Singh D, Anand PJ, Fleisher AS, Mata J, et al. In vivo performance of a microfabricated catheter for intraparenchymal delivery. J Neurosci Methods (2014) 229:76-83. doi:10.1016/j.jneumeth.2014.03.016

24. O'Cathail SM, Pokrovska TD, Maughan TS, Fisher KD, Seymour LW, Hawkins MA. Combining oncolytic adenovirus with radiation-a paradigm for the future of radiosensitization. Front Oncol (2017) 7:153. doi:10.3389/ fonc. 2017.00153

Conflict of Interest Statement: The authors declare that the research was conducted in the absence of any commercial or financial relationships that could be construed as a potential conflict of interest.

Copyright (c) 2018 Tejada, Díez-Valle, Domínguez, Patiño-García, González-Huarriz, Fueyo, Gomez-Manzano, Idoate, Peterkin and Alonso. This is an open-access article distributed under the terms of the Creative Commons Attribution License (CC $B Y)$. The use, distribution or reproduction in other forums is permitted, provided the original author(s) and the copyright owner are credited and that the original publication in this journal is cited, in accordance with accepted academic practice. No use, distribution or reproduction is permitted which does not comply with these terms. 